\title{
The importance of the cerebroplacental ratio for the prognosis of neonatal outcome in AGA fetuses
}

\section{Luisa Mecke ( $\boldsymbol{\nabla}$ Luisa.mecke@web.de)}

Otto-von-Guericke-University Magdeburg: Otto von Guericke Universitat Magdeburg https://orcid.org/0000-0002-1041-8828

\section{Atanas Ignatov \\ Anke Redlich}

\section{Research Article}

Keywords: cerebroplacental ratio, CPR, Doppler ultrasound, AGA, neonatal outcome

Posted Date: February 9th, 2022

DOI: https://doi.org/10.21203/rs.3.rs-1332918/v1

License: (c) (i) This work is licensed under a Creative Commons Attribution 4.0 International License.

Read Full License 
The importance of the cerebroplacental ratio for the prognosis of neonatal outcome in AGA fetuses

\section{Mecke' ${ }^{1}$ A. Ignatov ${ }^{1}$, A. Redlich ${ }^{1}$}

${ }^{1}$ Otto von Guericke University, University Clinic for Gynaecology, Obstetrics and Reproductive Medicine, Magdeburg, Germany

\section{Correspondence:}

Luisa Mecke

Luisa.mecke@web.de

ORCID iD: 0000-0002-1041-8828

Tel. +4917694874484

\section{Acknowledgements}

Financial support was provided by the Medical Faculty of Otto von Guericke University Magdeburg, Germany, in the form of a doctoral scholarship as part of the performance-oriented granting of funding. 


\section{Introduction}

Neonatal outcome is routinely assessed by sonographic fetal weight estimation and cardiotocography (CTG). Doppler sonography has been established as a safe method for assessing the fetal blood supply during pregnancy. Indications for a Doppler sonographic examination are mainly in high-risk pregnancies. As a Doppler sonographic parameter, the cerebroplacental ratio (CPR) provides information about fetal haemodynamics as well as intrauterine hypoxic states. With the help of the CPR, statements can be made about a redistribution of the fetal blood volume in response to a metabolic change. The CPR is defined by the quotient of the Doppler indices of the fetal middle cerebral artery (MCA) and the umbilical artery (UA). The Pulsatility Index (PI) is usually used for this purpose[1]. If the fetus is in a hypoxic state or growth retardation, the cerebral vessels dilate to maintain blood flow to the brain ("brain-sparing effect")[2]. This increased end-diastolic blood flow velocity is reflected in decreased Doppler indices (PI) of the MCA. Furthermore, the placental blood flow resistance increases and the end-diastolic blood flow velocity in the umbilical vessels decreases[1]. This results in increased Doppler indices of the UA. As a product of this change in perfusion, CPR decreases. CPR was first described by Arbeille et al. in 1987. In their study of 40 normal pregnancies, they showed that the cerebral vascular resistance is higher than the resistance within the placental vessels and that CPR is therefore $>1$ throughout pregnancy[3]. Furthermore, CPR showed a higher sensitivity than either Doppler index (UA, MCA) alone. Since then, CPR has been the subject of numerous scientific studies[4-7].

The aim of this scientific study is to determine to what extent CPR can be integrated into routine obstetric examinations as a valid parameter for assessing fetal status. In appropriate for gestational age (AGA) fetuses the potential value of CPR to assess neonatal outcome and accurately predict a secondary caesarean section due to fetal distress was investigated. For this purpose, retrospectively collected data from the intensive pregnancy consultation (IPC) of the University Women's Clinic Magdeburg, Germany, were analyzed.

\section{Methods}

The present work is a retrospective, unicentre observational study. It has been approved by the Ethics Committee of the Medical Faculty of Otto von Guericke University Magdeburg. Data from all patients who had taken part in an examination as part of the intensive pregnancy consultation (IPC) at the University Women's Clinic of Otto von Guericke University Magdeburg in 2016 and 2017 were included $(n=1,739)$. Most of the patients attending this outpatient consultation decided to give birth at the University Women's Clinic, so that 
outpatient and inpatient data could be correlated with each other. The aim of the data collection was to combine the data from the outpatient consultation (IPC) with the corresponding data from the birth and to evaluate them with regard to the neonatal outcome. Sonographic examinations were performed with the aid of the Voluson S8 ultrasound system (GE Healthcare) using an abdominal transducer. Doppler sonography was used to determine the Doppler indices of the UA and MCA. CPR was then calculated. Microsoft Excel was used for data collection and processing. The most important factor for inclusion in the study was the presence of the PI of the UA as well as the PI of the MCA, as these two parameters are required for the determination of the CPR. In this study, a decreased CPR $<1.08$ is considered as pathological. Some patients underwent multiple examinations during the course of their pregnancy. In this case, the data of the examination with the lowest CPR in each case were included in the considerations in the sense of standardisation. Multiple pregnancies were excluded from the study. Cases with births outside the University Women's Clinic were also excluded, as there was no access to perinatal data for these. Of 1,739 patients who visited the IPC in 2016 and 2017, 800 pregnancies could be included in the observations according to the inclusion and exclusion criteria mentioned above (Fig. 1). These included 710 AGA fetuses and 90 SGA fetuses. The cut-off value for classification as an AGA fetus was an estimated fetal weight $\geq 10$ th percentile using Hadlock III formula[8, 9]. Large for gestational age (LGA) fetuses with an estimated weight above the 90th percentile were grouped with AGA fetuses. Of the 710 AGA fetuses, 669 had normal and 41 decreased prenatal CPR. AGA fetuses with normal and decreased CPR were compared in terms of neonatal outcome as well as mode of delivery (secondary caesarean section due to fetal distress). In another study arm, the same procedure was followed for the group of SGA fetuses. Poor neonatal outcome was associated with the following parameters: low birth weight, birth weight $<10$ th percentile, 1 -minute Apgar-score $<$ 7, 5-minute Apgar-score < 7, 10-minute Apgar-score $<$ 7, umbilical artery $\mathrm{pH}<7.20$, umbilical artery base excess $<-8.0$, transfer to neonatology, prolonged length of neonatology stay, presence of severe morbidity or mortality. Of all cases, no newborn had a 10minute Apgar-score < 7, so this parameter was omitted from the analysis. Likewise, neither intrauterine foetal death (IUFD) nor neonatal mortality occurred during the inpatient stay after birth. The presence of severe morbidity was defined as the presence of any of the following conditions: Respiratory Distress Syndrome (RDS), Intraventricular Haemorrhage (IVH), Periventricular Leukomalacia (PVL), Necrotising Enterocolitis (NEC), Retinopathia praematurorum (ROP), Bronchopulmonary Dysplasia (BPD) and sepsis. Secondary caesarean section due to fetal distress refers to delivery by caesarean section due to vital risk to the fetus. 
Signs of this danger are a pathological CTG or Doppler examination or an abnormal micro blood test. The statistical analysis was carried out with the support of the Institute for Biometry and Medical Informatics of the Medical Faculty of Otto von Guericke University Magdeburg. First, the CPR was calculated for all cases. Subsequently, the dataset was processed and transferred from Microsoft Excel to the statistical and analysis software IBM SPSS. For the evaluation, mainly non-parametric tests such as the Mann-Whitney $U$ test and the chi-square test were used. The significance level $\alpha$ was set at 0.05 (5\%). Means and standard deviations as well as percentages were rounded to the first decimal place.

\section{Results}

The examinations were performed between the $22 \mathrm{nd}$ and $42 \mathrm{nd}$ week of pregnancy. The majority of the examinations were carried out in the last two months of pregnancy. The median maternal age was 30.3 years (ranged 15 to 46 years).

Of the total population, 710 cases were classified as AGA fetuses using the Hadlock III formula for fetal weight estimation. Of these, 669 fetuses had a normal CPR and 41 had a decreased CPR. These two groups were compared on the basis of the individual parameters associated with neonatal outcome. In addition, the mode of delivery was included here in terms of a secondary caesarean section due to fetal distress.

The estimated fetal weight of the AGA fetuses was on average at the 45th percentile. In the group of AGA fetuses, $5.8 \%$ (41/710) presented a decreased CPR. The mean value of the CPR values determined was 1.86. Spontaneous delivery occurred in $63.1 \%$ (448/710) of AGA fetuses. Further $15.6 \%(111 / 710)$ of the patients underwent a primary caesarean section, $10.1 \%$ (72/710) underwent a secondary caesarean section for fetal distress and $11.1 \%$ (79/710) got a secondary caesarean section due to other (maternal) indications.

In the group of AGA fetuses with normal CPR, $9.0 \%$ (60/669) of cases underwent a caesarean section due to fetal distress (Table 1). Notably, in the AGA fetuses with pathological CPR, more than threefold the number $(29.3 \% ; 12 / 41)$ of caesarean sections had to be performed due to fetal distress. Therefore, abnormally low CPR in AGA fetuses was significantly associated with an increased rate of section due to fetal distress $(p<0,001)$.

On average, the birth weight of AGA fetuses with a normal CPR was 3,456 g, while fetuses with a decreased CPR weighed an average of 3,044 g after birth $(p<0.001$, Fig. 2). Thus, normal-weight fetuses with reduced CPR had an average birth weight that was approximately $12 \%$ lower than fetuses with normal CPR. Fetuses with decreased CPR were over five times more likely to have an estimated fetal weight below the 10 th percentile $(26.8 \% ; 11 / 41)$ than 
fetuses with normal CPR ( $p<0.001$, Table 2). In this group, only $5.1 \%(34 / 669)$ of the fetuses had an estimated fetal weight below the 10th percentile. At $26.8 \%$ (11/41), fetuses with reduced CPR had to be transferred to neonatology more than twice as often as fetuses with normal CPR $(11.2 \% ; 75 / 669, p=0.011)$. Also, with 7.4 days they had an almost sevenfold longer average length of stay in neonatology than the comparison group with only 1.1 days $(p=0.001)$. Severe morbidity was diagnosed in $4.9 \%(2 / 41)$ of fetuses with decreased CPR. In the group of fetuses with normal CPR, there were only $0.3 \%(2 / 669)$ cases with severe disease $(p=0.018)$. The two groups did not differ significantly in Apgar scores, umbilical artery $\mathrm{pH}$ and base excess. In conclusion, decreased CPR predicted fetal impairment correctly in five neonatal outcome parameters.

\section{Discussion}

\section{Limitations}

Limitations of the present study exist in particular with regard to the retrospective study design. In a prospective setting, patient data could have been followed directly and, if necessary, additional parameters that turned out to be relevant during the course could have been recorded and analysed. Furthermore, this would possibly have led to more far-reaching statements with regard to the planning of appropriate birth management. Another limitation is the relatively small number of cases included, 710 AGA fetuses. A larger study population might make the results more valid and representative. Definitions and classifications are of great importance within this work. For example, there are no uniform thresholds and specifications for CPR, estimated fetal weight and neonatal outcome. For CPR, there are cut-off values of $<1,<1.08$ or $<5$ th percentile[1]. The boundary between AGA and SGA fetuses can be drawn according to an estimated fetal weight below the 3rd, 5th or 10th percentile. Furthermore, changes in outcomes may result from combining AGA and LGA fetuses. Neonatal outcome is also not a uniformly defined term. In order to quantify the condition of the child after birth, numerous authors use measurable parameters (e.g. birth weight, length of stay in neonatology). Attempts are sometimes made to measure the overall outcome of newborns with the help of composite scores, which combine various parameters. However, there is no scientific evidence for this type of score. It is also striking that significance with regard to an unfavourable neonatal outcome is not found in the Apgar score or in parameters of the fetal acid-base status. The results cast doubt on the significance of the two parameters mentioned with regard to fetal condition diagnostics. In particular, the Apgar score has limitations with regard to subjective factors in the assessment, interobserver variability and the dependence of the score on maternal 
anaesthesia, congenital malformations, gestational age, birth trauma and fetal immaturity[10]. Consequently, it is of great importance not to consider the parameters for the fetal outcome in isolation from each other, but to assess them in their overall context. The patients included in this study were examined at different times during pregnancy. CPR values were collected from the 22nd - 42nd week of gestation in the included cases. Since CPR changes in the course of pregnancy and no distinction was made here according to the time of examination, this can lead to impaired comparability. The good interrater reliability in this study should be positively emphasised, as the examinations in the context of IPC were carried out by one and the same specialist. Since sonography is often very examiner-dependent, independence from the examiner proves to be a clear advantage in this scientific observation.

\section{Value of CPR in AGA fetuses}

In AGA fetuses, CPR was shown in the present study to be a reliable predictor of an impaired neonatal outcome in terms of a lower or below 10th percentile birth weight, transfer to neonatology, longer length of stay in neonatology and the presence of severe morbidity. Compared to the Apgar score and values of the infant blood gas analysis, these parameters seem to provide more precise information about the neonatal outcome. Furthermore, a CPR $<1.08$ is significantly associated with an increased risk of caesarean section due to fetal distress. The majority of current studies and recommendations for everyday clinical practice so far refers to the recording of CPR in risk groups, e.g. IUGR fetuses[11-13]. It is therefore striking that, according to the results of the present study, CPR appears to have such great significance in AGA fetuses. In 2013, Prior et al. published a prospective study in which Doppler sonographic examinations were performed on 400 AGA pregnancies immediately before birth. Of the fetuses delivered by caesarean section due to fetal compromise, $36.4 \%$ had a CPR $<10$ th percentile antenatally compared with only $9.5 \%$ who had a CPR $\geq 10$ th percentile $(p<0.001)[14]$. No fetus with a CPR $>90$ th percentile had a need for caesarean section due to fetal compromise. Furthermore, fetuses with pathologically low CPR showed compromise in terms of significantly higher rates of meconium-stained amniotic fluid $(p=0.02)$ and CTG abnormalities $(p<0.001)[14]$. In terms of neonatal outcome, fetuses with normal CPR were born with significantly higher birth weight percentiles $(p=0.04)$. These data demonstrate the high significance of CPR in identifying intrapartum risk in AGA fetuses and suggest the use of this parameter for prenatal risk stratification. Contrasting results are provided by a recent study by Buca et al.[15]. This prospective study included 553 AGA fetuses in the 37th -38 th pregnancy week. In pregnancies with and without perinatal morbidity, there were no significant 
differences between the mean values for PI of UA $(p=0.486)$ or MCA $(p=0.621)$, nor for CPR $(p=0.832$ ). In pregnancies with intrapartum complications, the mean PI of MCA was significantly lower than in the control group without complications $(1.47 \pm 0.4$ vs. $1.61 \pm 0.4$; $p=0.0039$ ), while there were no differences in the other Doppler parameters including CPR $(p=0.108)[15]$. Furthermore, none of the Doppler parameters studied correlated with abnormal acid-base status at birth. Similar results were previously reported in a prospective study by Akolekar et al. in 2019[16]. The significance of CPR during a routine examination in the 37th - 38th week of pregnancy was investigated on a prospective basis in over 47,000 pregnancies. A CPR $<10$ th percentile was associated with an unfavourable perinatal outcome, perinatal hypoxia, caesarean delivery due to fetal risk and a birth weight below the 3rd percentile[16]. However, multivariate regression analysis showed that the prediction of these adverse events was only marginally enhanced by the addition of CPR to the commonly used maternal history. Furthermore, the detection rates for individual adverse perinatal events were relatively unreliable at 13-26\% and a false positive rate of about $10 \%[16]$. According to this study, CPR does not provide any additional diagnostic advantage in the cohort of AGA fetuses as well as in the group of SGA fetuses. In contrast, according to the study by Khalil et al. mentioned in the previous section, lower CPR is associated with the need for surgical delivery due to fetal compromise and with admission to neonatology, regardless of the size of the fetus[17]. Overall, the scientific literature contains conflicting results on the use of CPR as a diagnostic option in AGA fetuses. The data and results of the present study confirm that CPR is highly predictive of impaired neonatal outcome in the cohort of AGA fetuses, which could be used for routine screening in the low-risk population in the future.

\section{Conclusion}

The data demonstrate that decreased CPR in AGA fetuses correlates with impaired neonatal outcome in terms of a lower birth weight, transfer to neonatology, longer length of stay in neonatology and the presence of severe morbidity as well as secondary caesarean section due to fetal distress. Overall, the results are promising for the future integration of CPR into a reliable obstetric screening. This will require further, especially prospective, studies. Further investigations of the present study will evaluate the informative value of CPR in SGA fetuses and compare CPR with previously established screening methods such as estimated fetal weight and CTG. 


\section{References}

1. DeVore GR (2015) The importance of the cerebroplacental ratio in the evaluation of fetal well-being in SGA and AGA fetuses. Am J Obstet Gynecol 213:5-15. https://doi.org/10.1016/j.ajog.2015.05.024

2. Stampalija T, Arabin B, Wolf $\mathrm{H}$ et al. (2017) Is middle cerebral artery Doppler related to neonatal and 2-year infant outcome in early fetal growth restriction? Am J Obstet Gynecol:521.e1-521.e13. https://doi.org/10.1016/j.ajog.2017.01.001

3. Arbeille PH, Roncin A, Berson M et al. (1987) Exploration of the fetal cerebral blood flow by duplex doppler-linear array system in normal and pathological pregnancies. Ultrasound in Medicine \& Biology 13:329-337. https://doi.org/10.1016/03015629(87)90166-9

4. Bahado-Singh RO, Kovanci E, Jeffres A et al. (1999) The Doppler cerebroplacental ratio and perinatal outcome in intrauterine growth restriction. Am J Obstet Gynecol 180:750 756. https://doi.org/10.1016/s0002-9378(99)70283-8

5. Baschat AA, Gembruch U (2003) The cerebroplacental Doppler ratio revisited. Ultrasound in obstetrics \& gynecology: the official journal of the International Society of Ultrasound in Obstetrics and Gynecology 21:124-127. https://doi.org/10.1002/uog.20

6. Odibo AO, Riddick C, Pare E et al. (2005) Cerebroplacental Doppler ratio and adverse perinatal outcomes in intrauterine growth restriction: evaluating the impact of using gestational age-specific reference values. Journal of ultrasound in medicine: official journal of the American Institute of Ultrasound in Medicine 24:1223-1228. https://doi.org/10.7863/jum.2005.24.9.1223

7. Ebbing C, Rasmussen S, Kiserud T (2007) Middle cerebral artery blood flow velocities and pulsatility index and the cerebroplacental pulsatility ratio: longitudinal reference ranges and terms for serial measurements. Ultrasound in obstetrics \& gynecology: the official journal of the International Society of Ultrasound in Obstetrics and Gynecology 30:287-296. https://doi.org/10.1002/uog.4088

8. Hadlock FP, Harrist RB, Carpenter RJ et al. (1984) Sonographic estimation of fetal weight. The value of femur length in addition to head and abdomen measurements. Radiology 150:535-540. https://doi.org/10.1148/radiology.150.2.6691115

9. Hadlock FP, Harrist RB, Sharman RS et al. (1985) Estimation of fetal weight with the use of head, body, and femur measurements--a prospective study. Am J Obstet Gynecol 151:333-337. https://doi.org/10.1016/0002-9378(85)90298-4 
10. American College of Obstetricians and Gynecologists Commitee (2015) The Apgar Score. Obstet Gynecol:126.e52-e55. https://doi.org/10.1542/peds.2015-2651

11. Flood K, Unterscheider J, Daly S et al. (2014) The role of brain sparing in the prediction of adverse outcomes in intrauterine growth restriction: results of the multicenter PORTO Study. Am J Obstet Gynecol:288.e1-5. https://doi.org/10.1016/j.ajog.2014.05.008

12. Figueras F, Savchev S, Triunfo S et al. (2015) An integrated model with classification criteria to predict small-for-gestational-age fetuses at risk of adverse perinatal outcome. Ultrasound in obstetrics \& gynecology: the official journal of the International Society of Ultrasound in Obstetrics and Gynecology:279-285. https://doi.org/10.1002/uog.14714

13. Cruz-Martínez R, Figueras F, Hernandez-Andrade E et al. (2011) Fetal brain Doppler to predict cesarean delivery for nonreassuring fetal status in term small-for-gestational-age fetuses. Obstet Gynecol 117:618-626. https://doi.org/10.1097/AOG.0b013e31820b0884

14. Prior T, Mullins E, Bennett P et al. (2013) Prediction of intrapartum fetal compromise using the cerebroumbilical ratio: a prospective observational study. Am J Obstet Gynecol:124.e1-6. https://doi.org/10.1016/j.ajog.2012.11.016

15. Buca D, Rizzo G, Gustapane S et al. (2021) Diagnostische Genauigkeit der Dopplersonografie zur Vorhersage des perinatalen Outcomes bei reifgeborenen Föten: Eine prospektive Studie (Diagnostic Accuracy of Doppler Ultrasound in Predicting Perinatal Outcome in Appropriate for Gestational Age Fetuses: A Prospective Study). Ultraschall in Med 42:404-410. https://doi.org/10.1055/a-1072-5161

16. Akolekar R, Ciobanu A, Zingler E et al. (2019) Routine assessment of cerebroplacental ratio at 35-37 weeks' gestation in the prediction of adverse perinatal outcome. Am J Obstet Gynecol 221:65.e1-65.e18. https://doi.org/10.1016/j.ajog.2019.03.002

17. Khalil AA, Morales-Rosello J, Morlando M et al. (2015) Is fetal cerebroplacental ratio an independent predictor of intrapartum fetal compromise and neonatal unit admission? Am J Obstet Gynecol:54.e1-54.e10. https://doi.org/10.1016/j.ajog.2014.10.024 


\section{Statements and Declarations}

\section{Funding}

Financial support was provided by the Medical Faculty of Otto von Guericke University Magdeburg, Germany, in the form of a doctoral scholarship as part of the performance-oriented granting of funding.

\section{Competing Interests}

The authors have no relevant financial or non-financial interests to disclose.

\section{Author Contribution}

L. Mecke: study conception, data collection, discussion of results, manuscript writing

A. Ignatov: project administration, discussion of results, manuscript editing

A. Redlich: project development and administration, discussion of results, manuscript editing

\section{Ethics approval}

This study was performed in line with the principles of the Declaration of Helsinki. Approval was granted by the Ethics Committee of Otto von Guericke University Magdeburg, Germany.

\section{Consent to participate}

Informed consent was obtained from all individual participants included in the study.

\section{Consent to publish}

The manuscript does not contain any individual person's data in any form. 
Table 1 Association of cerebroplacental ratio with secondary caesarean section due to fetal distress in 710 AGA fetuses

\begin{tabular}{|l|l|l|l|}
\hline & $\begin{array}{l}\text { Normal CPR } \geq 1.08 \\
(\mathrm{n}=669)\end{array}$ & $\begin{array}{l}\text { Decreased CPR }<1.08 \\
(\mathrm{n}=41)\end{array}$ & $p$-value \\
\hline $\begin{array}{l}\text { Secondary caesarean } \\
\text { section for fetal distress }\end{array}$ & $\mathbf{6 0}(\mathbf{9 . 0} \%)$ & $\mathbf{1 2}(\mathbf{2 9 . 3} \%)$ & $<\mathbf{0 . 0 0 1}^{\mathrm{a}}$ \\
\hline
\end{tabular}

Data are given as n (\%). Paired comparison: ${ }^{a}$ Fisher's exact test. CPR: Cerebroplacental ratio. 
Table 2 Association of cerebroplacental ratio with neonatal outcome in 710 AGA fetuses

\begin{tabular}{|c|c|c|c|c|}
\hline & & $\begin{array}{l}\text { Normal CPR } \\
\geq 1.08 \\
(\mathrm{n}=669)\end{array}$ & $\begin{array}{l}\text { Decreased CPR } \\
<1.08 \\
(\mathrm{n}=41)\end{array}$ & $p$-value \\
\hline \multirow{9}{*}{ 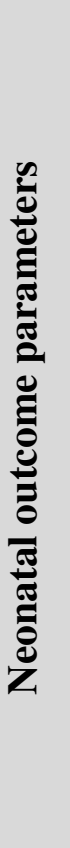 } & Birth weight $[\mathrm{g}]$ & $3,455.7 \pm 512.0$ & $3,044.0 \pm 691.2$ & $<0.001^{\mathrm{b}}$ \\
\hline & $\begin{array}{l}\text { Birth weight } \\
<10 \text { th percentile }\end{array}$ & $34(5.1 \%)$ & $11(26.8 \%)$ & $<0.001^{\mathrm{a}}$ \\
\hline & 1-min Apgar score $<7$ & $14(2.1 \%)$ & $1(2.4 \%)$ & $1.000^{\mathrm{a}}$ \\
\hline & 5-min Apgar score $<7$ & $1(0.1 \%)$ & $0(0.0 \%)$ & $1.000^{\mathrm{a}}$ \\
\hline & $\begin{array}{l}\text { Umbilical artery } \mathrm{pH} \text { at } \\
\text { delivery }<7.20\end{array}$ & $92(14.2 \%)$ & $4(10.0 \%)$ & $0.461^{\mathrm{c}}$ \\
\hline & Base excess $<-8.0$ & $46(7.1 \%)$ & $1(2.5 \%)$ & $0.511^{\mathrm{a}}$ \\
\hline & $\begin{array}{l}\text { Admission to } \\
\text { neonatology }\end{array}$ & $75(11.2 \%)$ & $11(26.8 \%)$ & $0.011^{\mathrm{a}}$ \\
\hline & $\begin{array}{l}\text { Length of stay in } \\
\text { neonatology [days] }\end{array}$ & $1.1 \pm 5.5$ & $7.4 \pm 25.5$ & $0.001^{b}$ \\
\hline & Severe morbidity* & $2(0.3 \%)$ & $2(4.9 \%)$ & $0.018^{\mathrm{a}}$ \\
\hline
\end{tabular}

Data are given as mean \pm SD or n (\%). Paired comparisons: ${ }^{b}$ Mann-Whitney U test, ${ }^{c}$ Pearson's chi-square test, 'Fisher's exact test. *Severe morbidity: presence of any of the following: Respiratory Distress Syndrome (RDS), Intraventricular Haemorrhage (IVH), Periventricular Leukomalacia (PVL), Necrotising Enterocolitis (NEC), Retinopathia praematurorum (ROP), Bronchopulmonary Dysplasia (BPD), sepsis. CPR: Cerebroplacental ratio. 


\section{Figure captions}

Fig. 1 Flowchart of the data collection process.

CPR: Cerebroplacental ratio, AGA: Appropriate for gestational age, SGA: Small for gestational age

Fig. 2 Boxplot comparing mean birth weight of AGA fetuses with normal and decreased CPR.

CPR: Cerebroplacental ratio 
Figures

\section{Intensive Pregnancy Consultation of University Women's Clinic Magdeburg, Germany, 2016 and $2017(\mathrm{n}=1,739)$}

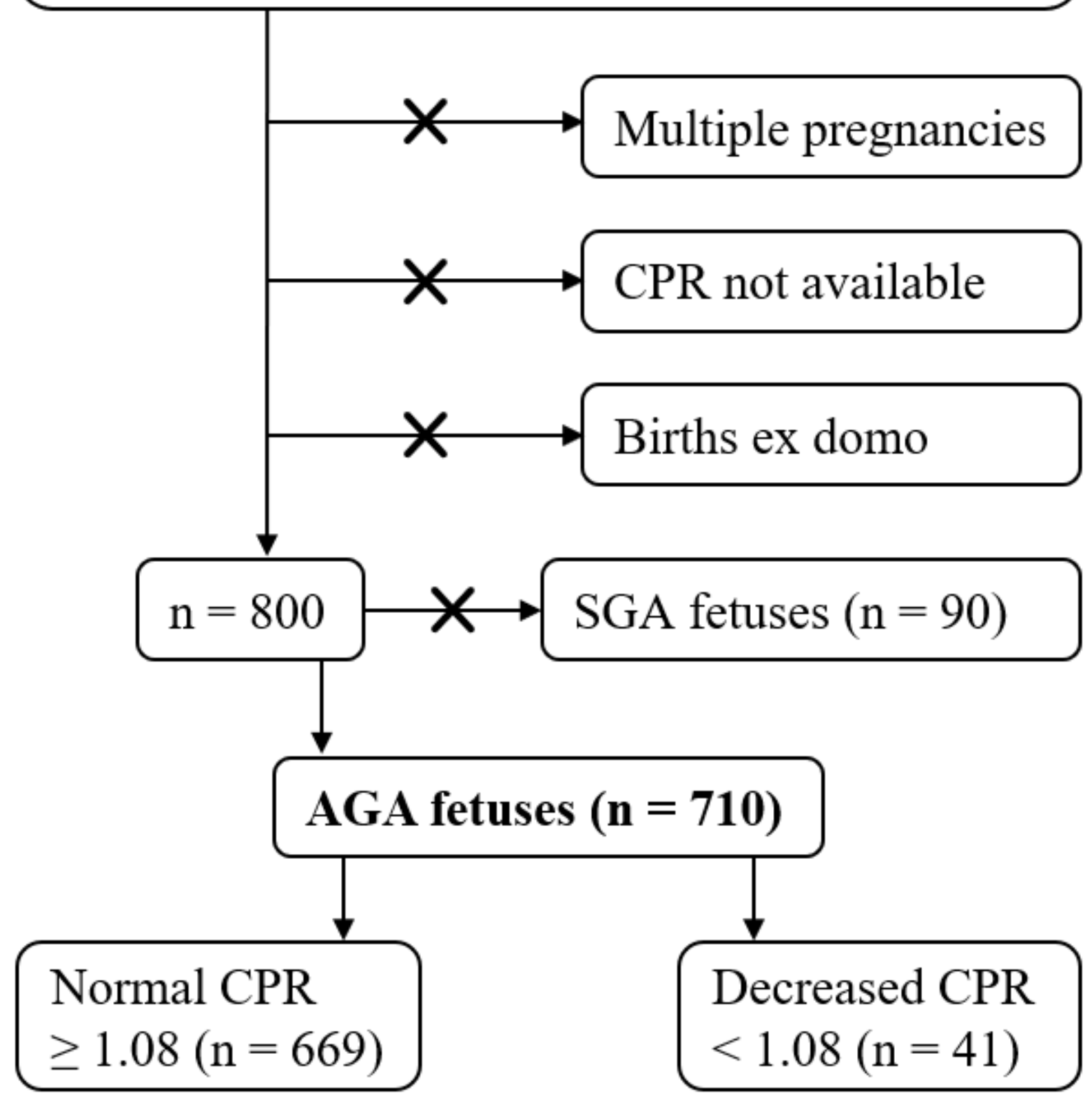

Figure 1

Flowchart of the data collection process.

CPR: Cerebroplacental ratio, AGA: Appropriate for gestational age, SGA: Small for gestational age 


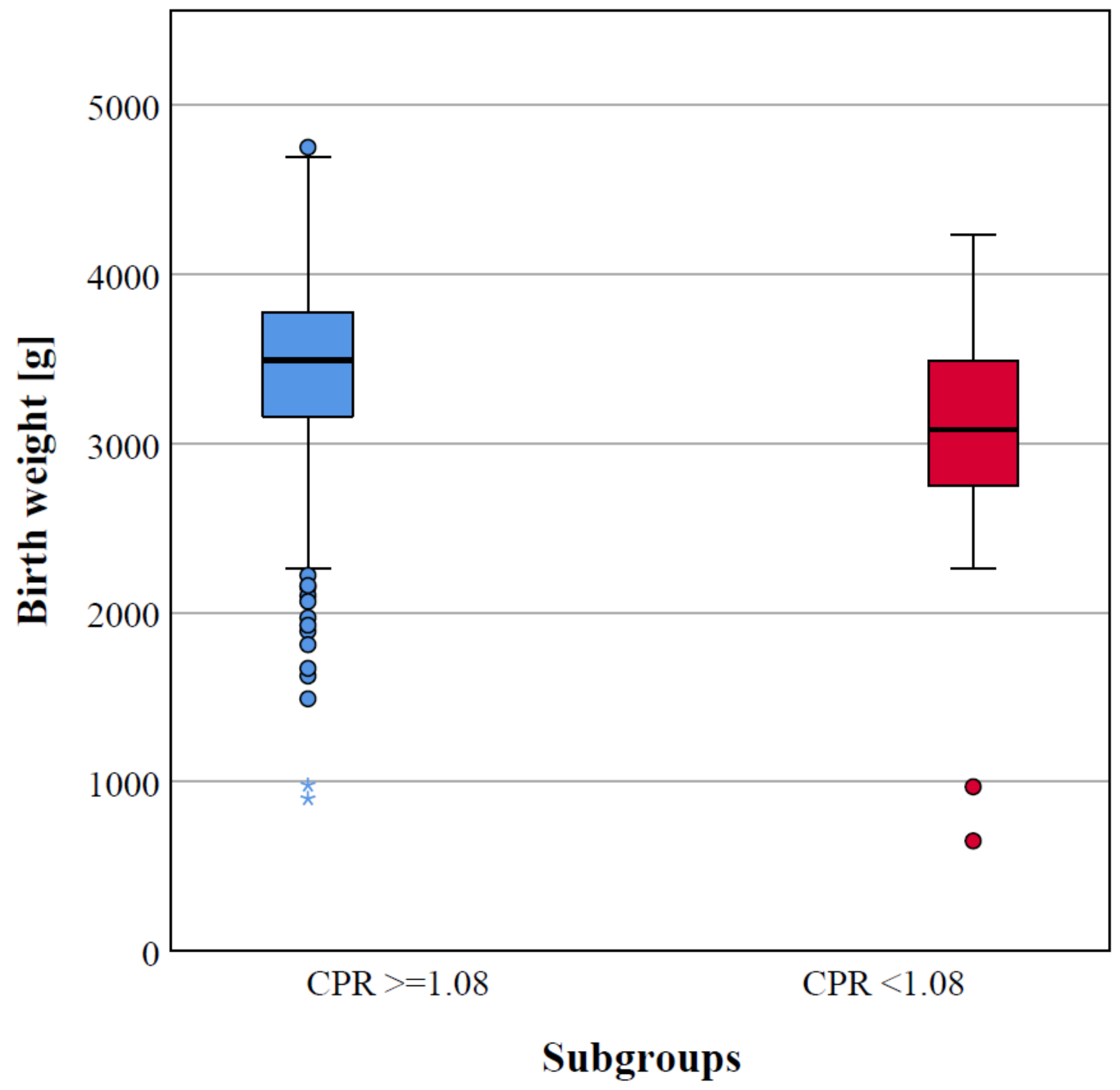

Figure 2

Boxplot comparing mean birth weight of AGA fetuses with normal and decreased CPR.

CPR: Cerebroplacental ratio 